\title{
Prevotella timonensis
}

National Cancer Institute

\section{Source}

National Cancer Institute. Prevotella timonensis. NCI Thesaurus. Code C124361.

A species of oblig ately anaerobic, non-pigmented, non-spore-forming, non-motile, Gram-negative straight rod-shaped bacteria in the family Prevotellaceae first isolated from a human breast abscess. P. timonensis is able to ferment glucose, lactose and maltose. 